\title{
A Fourth Order Difference Method For a Nonlinear Elliptic PDEs in Two Dimension Space
}

\author{
P. K. Pandey \\ Present address :Department of Information Technology ,College of Applied Sciences,Ministry of Higher Education, \\ P.Box No. 1905, PC-211, Salalah, Sultanate of Oman. \\ Department of Mathematics, Dyal Singh College (Univ. of Delhi), Lodhi Road, New Delhi-110003 , India \\ pramod_10p@hotmail.com

\begin{abstract}
In this article, we have presented a novel high order difference method for solving nonlinear elliptic equations with constant coefficients in two dimensions Cartesian coordinate system subject to Dirichlet boundary conditions. The present fourth order method based on the exponential techniques. The method reduces to central difference method when exponential function present in method linearized. We present numerical experiments to demonstrate the efficiency of the method and validity of our fourth order method.
\end{abstract}

Keywords: Exponential method, finite difference method, fourth order method,maximum absolute error, nonlinear elliptic equations.

MSC2010 65N06.

\section{Introduction and preliminaries}

In this article, we propose a finite difference method for solving nonlinear elliptic problems,

$$
\frac{\partial^{2} u}{\partial x^{2}}+\frac{\partial^{2} u}{\partial y^{2}}=f\left(x, y, u, u_{x}, u_{y}\right), \quad a \leq x, y \leq b
$$

in square region $\Omega=\{(x, y): a \leq x \leq b, a \leq y \leq b\}$ with the boundary conditions

$$
u(x, y)=g(x, y), \quad \text { on } \quad \partial \Omega
$$

where $\partial \Omega$ is boundary of $\Omega$.

There have been different approaches to solve these problems depends on the geometry of the domain. For irregular domain mostly preferred method is finite element whereas for regular domain it is finite difference method due to easy in implementation and d computational efficiency. The frequently used difference methods are central difference method and upwind difference method. These methods are known as standard finite difference method in the literature. There also exist several studies in the literature in which the standard finite difference method applied to solve elliptic PDEs in irregular domain 1, 22. But solving elliptic partial differential equations by the fourth order method difference method are attractive and have drawn the attention of the researchers to numerically solving elliptic problems either with variable or constant coefficients [3, 4] and references there in. To obtain more accurate numerical results several authors proposed improved finite difference method. Recently a compact 9-point fourth order off step finite difference concretization, for solving the system of nonlinear, variable coefficients PDEs and compact 9-point exponential difference method reported in [5, 6].

In this article, we generalize a 9-point exponential difference method for numerical solution of two dimensional general elliptic problems (1). The derivation of the method depends on difference approximation of the derivative 
on discrete mesh points in region of interest. We compare the performance of the method in reference with the other traditional methods in solving problems.

The present work is organized as follows .In section 2: we present novel exponential finite difference approximation for the general elliptic PDEs problem. A novel finite difference method is presented so that the resulting difference equation need satisfies the boundary conditions exactly. A derivation of the present method discussed in section 3 . A local truncation error and convergence of the method discussed in section 4 and 5 . Finally, the application of the developed method presented together with illustrative numerical results has been produced to show the efficiency of the method in section 6 . A discussion and conclusion on the performance of the method are presented in section 7 .

\section{The Exponential Finite Difference Method}

Consider the square domain $\Omega=[a, b] \times[a, b]$ for the solution of problem (1). Let $h=(b-a) \div N$ be the uniform mesh size in the $\mathrm{x}$ and $\mathrm{y}$ directions of the Cartesian coordinate system parallel to coordinate axes. Generate mesh points $\left(x_{i}, y_{j}\right), x_{i}=a \pm i h, i=0,1,2, \cdots N$ and $y_{j}=a \pm j h, i=0,1,2, \cdots N$. Let denote the central mesh point $\left(x_{i}, y_{j}\right)$ by $(i, j)$. Consider other mesh points $(i \pm 1, j),(i, j \pm 1)$ and $(i \pm 1, j \pm 1)$ neighbouring to the central mesh point $(i, j)$. These nine points together constitute a compact cell. Let us denote the exact and approximate values of the solution of the problem (1) at mesh point $(i, j)$ by $U_{i j}$ and $u_{i j}$ respectively. A nine point fourth order compact exponential difference method for the problem (1)is defined as follows ,

$$
\begin{gathered}
\bar{U}_{x i, j}=\frac{U_{i+1, j}-U_{i-1, j}}{2 h} \\
\bar{U}_{x i \pm 1, j}=\frac{ \pm 3 U_{i \pm 1, j} \mp 4 U_{i, j} \pm U_{i \mp 1, j}}{2 h} \\
\bar{U}_{x i, j \pm 1}=\frac{U_{i+1, j \pm 1}-U_{i-1, j \pm 1}}{2 h} \\
\bar{U}_{x x i, j \pm 1}=\frac{U_{i+1, j \pm 1}-2 U_{i, j \pm 1}+U_{i-1, j \pm 1}}{h^{2}} \\
\bar{U}_{y i, j}=\frac{U_{i, j+1}-U_{i, j-1}}{2 h} \\
\bar{U}_{y i, j \pm 1}=\frac{ \pm 3 U_{i, j \pm 1} \mp 4 U_{i, j} \pm U_{i, j \mp 1}}{2 h} \\
\bar{U}_{y i \pm 1, j}=\frac{U_{i \pm 1, j+1}-U_{i \pm 1, j-1}}{2 h} \\
\bar{U}_{y y i \pm 1, j}=\frac{U_{i \pm 1, j+1}-2 U_{i \pm 1, j}+U_{i \pm 1, j-1}}{h^{2}}
\end{gathered}
$$

Define

$$
\begin{gathered}
\bar{f}_{i \pm 1, j}=f\left(x_{i \pm 1}, y_{j}, U_{i \pm 1, j}, \bar{U}_{x i \pm 1, j}, \bar{U}_{y i \pm 1, j}\right) \\
\bar{f}_{i, j \pm 1}=f\left(x_{i}, y_{j \pm 1}, U_{i, j \pm 1}, \bar{U}_{x i, j \pm 1}, \bar{U}_{y i, j \pm 1}\right) \\
\overline{\bar{U}}_{x i, j}=\bar{U}_{x i, j}+a_{1} h\left(\bar{U}_{y y i+1, j}-\bar{U}_{y y i-1, j}\right)+a_{2} h\left(\bar{f}_{i+1, j}-\bar{f}_{i-1, j}\right) \\
\overline{\bar{U}}_{y i, j}=\bar{U}_{y i, j}+a_{3} h\left(\bar{U}_{x x i, j+1}-\bar{U}_{x x i, j-1}\right)+a_{4} h\left(\bar{f}_{i, j+1}-\bar{f}_{i, j-1}\right) \\
\widehat{\bar{U}}_{x i, j}=\bar{U}_{x i, j}+a_{5} h\left(\bar{U}_{y y i+1, j}-\bar{U}_{y y i-1, j}\right)+a_{6} h\left(\bar{f}_{i+1, j}-\bar{f}_{i-1, j}\right) \\
\widehat{\bar{U}}_{y i, j}=\bar{U}_{y i, j}+a_{7} h\left(\bar{U}_{x x i, j+1}-\bar{U}_{x x i, j-1}\right)+a_{8} h\left(\bar{f}_{i, j+1}-\bar{f}_{i, j-1}\right)
\end{gathered}
$$

where $a_{m}, m=1,2, \ldots, 7,8$ are free parameter to be determined.

Finally define,

$$
\begin{aligned}
& \overline{\bar{f}}_{i, j}=f\left(x_{i}, y_{j}, U_{i, j}, \overline{\bar{U}}_{x i, j}, \overline{\bar{U}}_{y i, j}\right) \\
& \widehat{\bar{f}}_{i, j}=f\left(x_{i}, y_{j}, U_{i, j}, \widehat{\bar{U}}_{x i, j}, \widehat{\bar{U}}_{y i, j}\right)
\end{aligned}
$$


and

$$
\begin{aligned}
4\left(U_{i+1, j}+U_{i-1, j}+U_{i, j+1}+U_{i, j-1}\right)+\left(U_{i+1, j+1}+U_{i-1, j+1}+U_{i-1, j+1}+U_{i-1, j-1}\right) & -20 u_{i, j} \\
& =6 h^{2} \overline{\bar{f}}_{i j} \exp \left(\frac{h^{2} \nabla^{2} \hat{f}_{i, j}}{12 \overline{\bar{f}}_{i, j}}\right)
\end{aligned}
$$

$f_{i j} \neq 0, \quad \forall i, j=1,2, \ldots \ldots, N$.

\section{Derivation of the Method}

The existence and uniqueness of the solution of the problem (1) is assumed. We have not considered any specific assumption on the source function $f$ to ensure existence and uniqueness of the solution. If we have a problem (1) in which source function is $f(x, y)$ instead of $f\left(x, y, u, u_{x}, u_{y}\right)$. The nine point fourth order compact difference method discussed and developed as [5],

$$
\begin{gathered}
4\left(U_{i+1, j}+U_{i-1, j}+U_{i, j+1}+U_{i, j-1}\right)+\left(U_{i+1, j+1}+U_{i-1, j+1}+U_{i+1, j-1}+U_{i-1, j-1}\right)-20 u_{i, j} \\
=6 h^{2} f_{i, j} \exp \left(\frac{f_{i+1, j}+f_{i-1, j}+f_{i, j+1}+f_{i, j-1}-4 f_{i, j}}{12 f_{i, j}}\right)
\end{gathered}
$$

To develop a fourth order difference method for problem (1) when $f\left(x, y, u, u_{x}, u_{y}\right)$ is source function ,we need following approximations.

Using Taylor series expansion about point $\left(x_{i \pm 1}, y_{j}\right)$,from(4)and(9)we have,

$$
\begin{gathered}
\bar{U}_{x i \pm 1, j}=U_{x i \pm 1, j}-\frac{h^{2}}{3} U_{x x x i, j} \mp \frac{h^{3}}{12} U_{x x x x i, j}+O\left(h^{4}\right) \\
\bar{U}_{y i \pm 1, j}=U_{y i \pm 1, j}+\frac{h^{2}}{6} U_{y y y i \pm 1, j}+O\left(h^{4}\right)
\end{gathered}
$$

Define $G_{i \pm 1, m}=\left(\frac{\partial f}{\partial u_{x}}\right)_{i \pm 1, j}, H_{i \pm 1, m}=\left(\frac{\partial f}{\partial u_{y}}\right)_{i \pm 1, j}$ and from(17-18),we find

$$
\bar{f}_{i \pm 1, j}=f_{i \pm 1, j}+\left(-\frac{h^{2}}{3} U_{x x x i, j} \mp \frac{h^{3}}{12} U_{x x x x i, j}\right) G_{i, j}+\frac{h^{2}}{6} U_{y y y i, j} H_{i, j}+O\left(h^{4}\right)
$$

Thus from (19), we have

$$
\begin{aligned}
\bar{f}_{i+1, j} & -\bar{f}_{i-1, j}=f_{i+1, j}-f_{i-1, j}-\frac{h^{3}}{6} U_{x x x x i, j} G i, j+O\left(h^{4}\right) \\
& =h\left(\frac{\partial f}{\partial x}\right)_{i, j}-\frac{h^{3}}{6} U_{x x x x i, j} G i, j+O\left(h^{4}\right) \\
& =h\left(U_{x x x i, j}+U_{x y y i, j}\right)-\frac{h^{3}}{6} U_{x x x x i, j} G i, j+O\left(h^{4}\right)
\end{aligned}
$$

Expand $(3,10)$ in Taylor series about $\left(x_{i}, y_{j}\right)$ and substitute in (11)together with (20), we have

$$
\overline{\bar{U}}_{x i, j}=U_{x i, j}+h^{2}\left(\frac{1}{6}+2 a_{2}\right) U_{x x x i, j}+2 h^{2}\left(a_{1}+a_{2}\right) U_{x y y i, j}+O\left(h^{4}\right)
$$

The approximation (21) will provide a fourth order approximation for $\overline{\bar{U}}_{i, j}$ if we have

$$
\begin{aligned}
& a_{1}+a_{2}=0 \\
& \frac{1}{6}+2 a_{2}=0
\end{aligned}
$$

Solving above system of equations in $a_{1}$ and $a_{2} \quad$, we have $a_{1}=\frac{1}{12} \quad$ and $a_{2}=\frac{-1}{12}$. Thus

$$
\overline{\bar{U}}_{x i, j}=\bar{U}_{x i, j}+\frac{h}{12}\left(\bar{U}_{y y i+1, j}-\bar{U}_{y y i-1, j}\right)-\frac{h}{12}\left(\bar{f}_{i+1, j}-\bar{f}_{i-1, j}\right)
$$


Similarly we have,

$$
\overline{\bar{U}}_{y i, j}=\bar{U}_{y i, j}+\frac{h}{12}\left(\bar{U}_{x x i, j+1}-\bar{U}_{x x i, j-1}\right)-\frac{h}{12}\left(\bar{f}_{i, j+1}-\bar{f}_{i, j-1}\right)
$$

From (22-23),we find that $\overline{\bar{U}}_{x i, j}$ and $\overline{\bar{U}}_{y i, j}$ respectively provides $O\left(h^{4}\right)$ approximation for $U_{x i, j}$ and $U_{y i, j}$ i.e.

$$
\begin{aligned}
& \overline{\bar{U}}_{x i, j}=U_{x i, j}+O\left(h^{4}\right) \\
& \overline{\bar{U}}_{y i, j}=U_{y i, j}+O\left(h^{4}\right)
\end{aligned}
$$

Thus from (24-25),we have

$$
\overline{\bar{f}}_{i, j}=f_{i, j}+O\left(h^{4}\right)
$$

Let us define

$$
h^{2} \nabla^{2} \hat{f}_{i, j}=\bar{f}_{i+1, j}+\bar{f}_{i-1, j}+\bar{f}_{i, j+1}+\bar{f}_{i, j-1}-4 \widehat{\bar{f}}_{i, j}
$$

Using above defined approximations, we can prove that $\bar{f}_{i+1, j}+\bar{f}_{i-1, j}+\bar{f}_{i, j+1}+\bar{f}_{i, j-1}-4 \widehat{\bar{f}}_{i, j}$ will provide fourth order approximation for $f_{i+1, j}+f_{i-1, j}+f_{i, j+1}+f_{i, j-1}-4 f_{i, j}$ if

$$
\widehat{\bar{U}}_{x i, j}=\bar{U}_{x i, j}+\frac{h}{8}\left(\bar{U}_{y y i+1, j}-\bar{U}_{y y i-1, j}\right)-\frac{h}{8}\left(\bar{f}_{i+1, j}-\bar{f}_{i-1, j}\right)
$$

and

$$
\widehat{\bar{U}}_{y i, j}=\bar{U}_{y i, j}+\frac{h}{8}\left(\bar{U}_{x x i, j+1}-\bar{U}_{x x i, j-1}\right)-\frac{h}{8}\left(\bar{f}_{i, j+1}-\bar{f}_{i, j-1}\right)
$$

Thus

$$
\bar{f}_{i+1, j}+\bar{f}_{i-1, j}+\bar{f}_{i, j+1}+\bar{f}_{i, j-1}-4 \widehat{\bar{f}}_{i, j}=f_{i+1, j}+f_{i-1, j}+f_{i, j+1}+f_{i, j-1}-4 f_{i, j}+O\left(h^{4}\right)
$$

From definition (27),finally we propose 9-point compact fourth order exponential difference method for numerically solving problem (1) as,

$$
\begin{gathered}
4\left(U_{i+1, j}+U_{i-1, j}+U_{i, j+1}+U_{i, j-1}\right)+\left(U_{i+1, j+1}+U_{i-1, j+1}+U_{i+1, j-1}+U_{i-1, j-1}\right)-20 u_{i, j} \\
=6 h^{2} \overline{\bar{f}}_{i j} \exp \left(\frac{\bar{f}_{i+1, j}+\bar{f}_{i-1, j}+\bar{f}_{i, j+1}+\bar{f}_{i, j-1}-4 \widehat{\bar{f}}_{i j}}{12 \overline{\bar{f}}_{i, j}}\right)
\end{gathered}
$$

If we write system of equations given by (31) at each mesh point, we will obtain either linear or nonlinear system of equations depends on source function $f$. To obtain a satisfactory solution of this system of equations with reasonable degree of accuracy, selection of the methods for solving this system of equations are very important.

\section{Local Truncation Error}

By Taylor series expansion of $f$ on mesh point $\left(x_{i}, y_{j}\right)$ and from $(26)$ and (30), we have

$$
\frac{\bar{f}_{i+1, j}+\bar{f}_{i-1, j}+\bar{f}_{i, j+1}+\bar{f}_{i, j-1}-4 \widehat{\bar{f}}_{i, j}}{12 \overline{\bar{f}}_{i j}}=\frac{h^{2}\left(\frac{\partial^{2} f}{\partial x^{2}}+\frac{\partial^{2} f}{\partial y^{2}}\right)_{i, j}+\frac{h^{4}}{12}\left(\frac{\partial^{4} f}{\partial x^{4}}+\frac{\partial^{4} f}{\partial y^{4}}\right)_{i, j}}{12 f_{i, j}}
$$

By expansion of exponential function up to three terms

$$
\begin{gathered}
\exp \left(\frac{h^{2}\left(\frac{\partial^{2} f}{\partial x^{2}}+\frac{\partial^{2} f}{\partial y^{2}}\right)_{i, j}+\frac{h^{4}}{12}\left(\frac{\partial^{4} f}{\partial x^{4}}+\frac{\partial^{4} f}{\partial y^{4}}\right)_{i, j}}{12 f_{i, j}}\right)=1+\frac{h^{2}\left(\frac{\partial^{2} f}{\partial x^{2}}+\frac{\partial^{2} f}{\partial y^{2}}\right)_{i, j}}{12 f_{i, j}}+\frac{h^{4}\left(\frac{\partial^{4} f}{\partial x^{4}}+\frac{\partial^{4} f}{\partial y^{4}}\right)_{i, j}}{144 f_{i, j}} \\
+\frac{h^{4}}{2}\left(\frac{\left(\frac{\partial^{2} f}{\partial x^{2}}+\frac{\partial^{2} f}{\partial y^{2}}\right)_{i, j}}{12 f_{i, j}}\right)^{2}+O\left(h^{6}\right) \\
=1+\frac{h^{2}}{12} \frac{U_{40 i, j}+2 U_{22 i, j}+U_{04 i, j}}{U_{20 i, j}+U_{02 i, j}}+\frac{h^{4}}{144} \frac{U_{60 i, j}+3 U_{42 i, j}+3 U_{24 i, j}+U_{06 i, j}}{U_{20 i, j}+U_{02 i, j}}
\end{gathered}
$$




$$
+\frac{h^{4}}{288} \frac{\left(U_{40 i, j}+2 U_{22 i, j}+U_{04 i, j}\right)^{2}}{\left(U_{20 i, j}+U_{02 i, j}\right)^{2}}+O\left(h^{6}\right)
$$

where $f_{i, j}=U_{x x i, j}+U_{y y i, j}, \ldots \ldots$..etc. Let $T_{i, j}$ be truncation error at mesh point $\left(x_{i}, y_{j}\right)$. By Taylor series expansion of $U$ at mesh point $\left(x_{i}, y_{j}\right)$, from (31) and (32) we have

$$
\begin{aligned}
& T_{i, j}= \frac{h^{6}}{1440}\left\{-12\left(3 U_{60 i, j}+5 U_{42 i, j}+5 U_{24 i, j}+3 U_{06 i, j}\right)-\frac{5\left(U_{40 i, j}+2 U_{22 i, j}+U_{04 i, j}\right)^{2}}{U_{20 i, j}+U_{02 i, j}}\right\}+O\left(h^{8}\right) \\
&\left|T_{i, j}\right| \leq \frac{h^{6}}{1440} \Phi+O\left(h^{8}\right)
\end{aligned}
$$

where $U_{m n i, j}=\left(\frac{\partial^{m+n} U}{\partial x^{m} \partial y^{n}}\right)_{i, j}, m, n=0,2,4,6$ and

$$
\Phi=\max _{2 \leq i, j \leq N}\left|-12\left(3 U_{60 i, j}+5 U_{42 i, j}+5 U_{24 i, j}+3 U_{06 i, j}\right)-\frac{5\left(U_{40 i, j}+2 U_{22 i, j}+U_{04 i, j}\right)^{2}}{U_{20 i, j}+U_{02 i, j}}\right| .
$$

\section{Convergence of the method}

We next discuss the convergence of the method and under suitable conditions prove that the order of the convergence is $O\left(h^{4}\right)$. For each $i, j=2(1) N$, let us define

$$
\begin{gathered}
6 h^{2} \overline{\bar{f}}_{i j} \exp \left(\frac{\bar{f}_{i+1, j}+\bar{f}_{i-1, j}+\bar{f}_{i, j+1}+\bar{f}_{i, j-1}-4 \widehat{\bar{f}}_{i j}}{12 \overline{\bar{f}}_{i, j}}\right) \\
\approx 6 h^{2} \overline{\bar{f}}_{i j}\left(1+\frac{\bar{f}_{i+1, j}+\bar{f}_{i-1, j}+\bar{f}_{i, j+1}+\bar{f}_{i, j-1}-4 \widehat{\bar{f}}_{i j}}{12 \overline{\bar{f}}_{i, j}}\right) \\
=\frac{h^{2}}{2}\left(12 \overline{\bar{f}}_{i j}+\bar{f}_{i+1, j}+\bar{f}_{i-1, j}+\bar{f}_{i, j+1}+\bar{f}_{i, j-1}-4 \widehat{\bar{f}}_{i j}\right) \\
\phi_{i, j}=\frac{h^{2}}{2}\left(12 \overline{\bar{f}}_{i j}+\bar{f}_{i+1, j}+\bar{f}_{i-1, j}+\bar{f}_{i, j+1}+\bar{f}_{i, j-1}-4 \widehat{\bar{f}}_{i j}\right)+\text { Boundary Values }
\end{gathered}
$$

and

$$
E_{i, j}=u_{i, j}-U_{i, j}, \quad 2 \leq i, j \leq N
$$

Let define matrix $\mathbf{S}_{(N-1)^{2} \times 1}$ in [6], as

$$
\mathbf{S}=\left[S_{2,2}, S_{3,2}, \ldots \ldots \ldots, S_{N, 2}, S_{2,3}, S_{3,3}, \ldots \ldots \ldots, S_{N, 3}, \ldots \ldots, S_{2, N}, S_{3, N}, \ldots \ldots, S_{N, N}\right]^{t}
$$

The the difference method (31)represents a system of nonlinear equations in the unknown $\quad u_{i, j}, 2 \leq i, j \leq N$. Using $S=\phi, u, U$ and $T$, we write (31) in matrix form as,

$$
\mathbf{D u}+\phi(\mathbf{u})=\mathbf{0}
$$

where $\mathbf{D}=[\mathbf{B A B}]_{(N-1)^{2} \times(N-1)^{2}}$ is tri block diagonal matrix, in which the tri diagonal matrics are $A=[-4,20,-4]_{(N-1)^{2} \times(N-1)^{2}}$ and $B=[-1,4,-1]_{(N-1)^{2} \times(N-1)^{2}}$. But $\mathbf{U}$ is exact solution of (31), which in matrix form can be written as

$$
\mathbf{D U}+\phi(\mathbf{U})+\mathbf{T}=\mathbf{0}
$$

where $\mathbf{T}$ is truncation error matrix and each element is of $O\left(h^{6}\right)$. Let us define

$$
\begin{gathered}
\bar{F}_{i \pm 1, j}=f\left(x_{i \pm 1}, y_{j}, U_{i \pm 1, j}, \bar{U}_{x i \pm 1, j}, \bar{U}_{y i \pm 1, j}\right) \\
\bar{f}_{i \pm 1, j}=f\left(x_{i \pm 1}, y_{j}, U_{i \pm 1, j}, \bar{u}_{x i \pm 1, j}, \bar{u}_{y i \pm 1, j}\right)
\end{gathered}
$$




$$
\begin{gathered}
\bar{F}_{i, j \pm 1}=f\left(x_{i}, y_{j \pm 1}, U_{i, j \pm 1}, \bar{U}_{x i, j \pm 1}, \bar{U}_{y i, j \pm 1}\right) \\
\bar{f}_{i, j \pm 1}=f\left(x_{i}, y_{j \pm 1}, U_{i, j \pm 1}, \bar{u}_{x i, j \pm 1}, \bar{u}_{y i, j \pm 1}\right) \\
\overline{\bar{F}}_{i, j}=f\left(x_{i}, y_{j}, U_{i, j}, \overline{\bar{U}}_{x i, j}, \overline{\bar{U}}_{y i, j}\right) \\
\overline{\bar{f}}_{i, j}=f\left(x_{i}, y_{j}, U_{i, j}, \overline{\bar{u}}_{x i, j}, \overline{\bar{u}}_{y i, j}\right) \\
\widehat{\bar{F}}_{i, j}=f\left(x_{i}, y_{j}, U_{i, j}, \widehat{\widehat{U}}_{x i, j}, \widehat{\widehat{U}}_{y i, j}\right) \\
\widehat{\bar{f}}_{i, j}=f\left(x_{i}, y_{j}, U_{i, j}, \widehat{\bar{u}}_{x i, j}, \widehat{\bar{u}}_{y i, j}\right)
\end{gathered}
$$

After linearization of $\bar{f}_{i \pm 1, j}$, we have,

$$
\bar{f}_{i \pm 1, j}-\bar{F}_{i \pm 1, j}=\left(u_{i \pm 1, j}-U_{i \pm 1, j}\right) G_{i \pm 1, j}+\left(\bar{u}_{x i \pm 1, j}-\bar{U}_{x i \pm 1, j}\right) H_{i \pm 1, j}+\left(\bar{u}_{y i \pm 1, j}-\bar{U}_{y i \pm 1, j}\right) I_{i \pm 1, j} .
$$

where $G=\frac{\partial f}{\partial U}, H=\frac{\partial f}{\partial \bar{U}_{x}}$ and $I=\frac{\partial f}{\partial \bar{U}_{y}}$.

Similarly we linearized and write for $\bar{f}_{i, j \pm 1}$ and $\overline{\bar{f}}_{i, j}$ and $\widehat{\bar{f}}_{i j}$ as,

$$
\begin{gathered}
\bar{f}_{i, j \pm 1}-\bar{F}_{i, j \pm 1}=\left(u_{i, j \pm 1}-U_{i, j \pm 1}\right) G_{i, j \pm 1}+\left(\bar{u}_{x i, j \pm 1}-\bar{U}_{x i, j \pm 1}\right) H_{i, j \pm 1}+\left(\bar{u}_{y i, j \pm 1}-\bar{U}_{y i, j \pm 1}\right) I_{i, j \pm 1} . \\
\overline{\bar{f}}_{i, j}-\overline{\bar{F}}_{i, j}=\left(u_{i, j}-U_{i, j}\right) G_{i, j}+\left(\overline{\bar{u}}_{x i, j}-\overline{\bar{U}}_{x i, j}\right) H_{i, j}^{1}+\left(\overline{\bar{u}}_{y i, j}-\overline{\bar{U}}_{y i, j}\right) I_{i, j}^{1} . \\
\widehat{\widehat{f}}_{i, j}-\widehat{\bar{F}}_{i, j}=\left(u_{i, j}-U_{i, j}\right) G_{i, j}+\left(\widehat{\bar{u}}_{x i, j}-\widehat{\bar{U}}_{x i, j}\right) H_{i, j}^{2}+\left(\widehat{\bar{u}}_{y i, j}-\widehat{\bar{U}}_{y i, j}\right) I_{i, j}^{2} .
\end{gathered}
$$

where $H^{1}=\frac{\partial f}{\partial \overline{\bar{U}}_{x}}, H^{2}=\frac{\partial f}{\partial \overline{\bar{U}}_{x}}, I^{1}=\frac{\partial f}{\partial \overline{\bar{U}}_{y}}$ and $I^{2}=\frac{\partial f}{\partial \overline{\bar{U}}_{y}}$.

By Taylor series expansions of $G_{i \pm 1, j}, G_{i, j \pm 1}, H_{i \pm 1, j}, H_{i, j \pm 1}, I_{i \pm 1, j}$ and $I_{i, j \pm 1}$ at mesh point $\left(x_{i}, y_{j}\right)$ and from (36)-(39), we get the matrix equation

$$
\phi(\mathbf{u})-\phi(\mathbf{U})=\mathbf{P E}
$$

where $\mathbf{P}=\left(P_{i, j}\right)_{(N-1)^{2} \times(N-1)^{2}}$ is the tri block diagonal matrix defined as,

$$
\begin{aligned}
& P_{(j-1)(N-1)+i,(j-1)(N-1)+i} \\
& =h^{2}\left\{6 G_{i, j}+H_{i, j}\left(2 H^{1}-H_{i, j}^{2}\right)+I_{i, j}\left(2 I^{1}-I_{i, j}^{2}\right)-2\left(H_{x i, j}+I_{y i, j}\right)\right\}+O\left(h^{3}\right) \\
& {[i=1(1) N-1, \quad j=1(1) N-1] .} \\
& P_{(j-1)(N-1)+i,(j-1)(N-1)+i+1} \\
& =\frac{h}{2}\left(H_{i, j}+4 H_{i, j}^{1}-H_{i, j}^{2}\right)+\frac{h^{2}}{2}\left(G_{i, j}+2 H_{x i, j}-H_{i, j}\left(2 H_{i, j}^{1}-H_{i, j}^{2}\right)\right)+O\left(h^{3}\right) \\
& {[i=1(1) N-2, \quad j=1(1) N-1] .} \\
& P_{(j-1)(N-1)+i,(j-1)(N-1)+i-1} \\
& =-\frac{h}{2}\left(H_{i, j}+4 H_{i, j}^{1}-H_{i, j}^{2}\right)+\frac{h^{2}}{2}\left(G_{i, j}+2 H_{x i, j}-H_{i, j}\left(2 H_{i, j}^{1}-H_{i, j}^{2}\right)\right)+O\left(h^{3}\right) \\
& {[i=2(1) N-1, \quad j=1(1) N-1] .} \\
& P_{(j-1)(N-1)+i,(j-2)(N-1)+i} \\
& =-\frac{h}{2}\left(I_{i, j}+4 I_{i, j}^{1}-I_{i, j}^{2}\right)+\frac{h^{2}}{2}\left(G_{i, j}+2 I_{y i, j}-I_{i, j}\left(2 I_{i, j}^{1}-I_{i, j}^{2}\right)\right)+O\left(h^{3}\right) \\
& {[i=1(1) N-1, \quad j=2(1) N-1] .}
\end{aligned}
$$




$$
\begin{aligned}
& P_{(j-1)(N-1)+i, j(N-1)+i} \\
& =\frac{h}{2}\left(4 I_{i, j}^{1}-I_{i, j}^{2}+I_{i, j}\right)+\frac{h^{2}}{2}\left(G_{i, j}+2 I_{y i, j}-I_{i, j}\left(2 I_{i, j}^{1}-I_{i, j}^{2}\right)\right)+O\left(h^{3}\right) \\
& {[i=1(1) N-1, \quad j=1(1) N-2] .} \\
& P_{(j-1)(N-1)+i,(j-2)(N-1)+i+1}=\frac{h}{2}\left(-2 I_{i, j}^{1}+I_{i, j}^{2}+2 H_{i, j}^{1}-H_{i, j}^{2}+H_{i, j}-I_{i, j}\right) \\
& +\frac{h^{2}}{4}\left(I_{i, j}\left(2 H_{i, j}^{1}-H_{i, j}^{2}\right)+H_{i, j}\left(2 I_{i, j}^{1}-I_{i, j}^{2}\right)-2\left(I_{x i, j}+H_{y i, j}\right)\right)+O\left(h^{3}\right) \\
& {[i=1(1) N-2, \quad j=2(1) N-1] .} \\
& P_{(j-1)(N-1)+i,(j-2)(N-1)+i-1}=\frac{h}{2}\left(-2 I_{i, j}^{1}+I_{i, j}^{2}-2 H_{i, j}^{1}+H_{i, j}^{2}-H_{i, j}-I_{i, j}\right) \\
& +\frac{h^{2}}{4}\left(I_{i, j}\left(-2 H_{i, j}^{1}+H_{i, j}^{2}\right)+H_{i, j}\left(-2 I_{i, j}^{1}+I_{i, j}^{2}\right)+2\left(I_{x i, j}+H_{y i, j}\right)\right)+O\left(h^{3}\right) \\
& {[i=2(1) N-1, \quad j=2(1) N-1] .} \\
& P_{(j-1)(N-1)+i, j(N-1)+i+1}=\frac{h}{2}\left(2 I_{i, j}^{1}-I_{i, j}^{2}+2 H_{i, j}^{1}-H_{i, j}^{2}+H_{i, j}+I_{i, j}\right) \\
& +\frac{h^{2}}{4}\left(I_{i, j}\left(-2 H_{i, j}^{1}+H_{i, j}^{2}\right)+H_{i, j}\left(-2 I_{i, j}^{1}+I_{i, j}^{2}\right)+2\left(I_{x i, j}+H_{y i, j}\right)\right)+O\left(h^{3}\right) \\
& {[i=1(1) N-2, \quad j=1(1) N-2] .} \\
& P_{(j-1)(N-1)+i, j(N-1)+i-1}=\frac{h}{2}\left(2 I_{i, j}^{1}-I_{i, j}^{2}-2 H_{i, j}^{1}+H_{i, j}^{2}-H_{i, j}+I_{i, j}\right) \\
& +\frac{h^{2}}{4}\left(I_{i, j}\left(2 H_{i, j}^{1}-H_{i, j}^{2}\right)+H_{i, j}\left(2 I_{i, j}^{1}-I_{i, j}^{2}\right)-2\left(I_{x i, j}+H_{y i, j}\right)\right)+O\left(h^{3}\right) \\
& {[i=2(1) N-1, \quad j=1(1) N-2] .}
\end{aligned}
$$

Let there are no roundoff errors in solution of difference equation (31), so from (34),(35) and (40) we get the error equation as

$$
(\mathbf{D}+\mathbf{P}) \mathbf{E}=\mathbf{T}
$$

Define a sets

$$
\begin{aligned}
& G_{0}=\left\{G_{i \pm 1, j}, G_{i, j \pm 1}, G_{i, j}\right\} \\
& H_{0}=\left\{H_{i \pm 1, j}, H_{i, j \pm 1}, H_{i, j}^{1}, H_{i, j}^{2}\right\} \\
& I_{0}=\left\{I_{i \pm 1, j}, I_{i, j \pm 1}, I_{i, j}^{1}, I_{i, j}^{2}\right\} \\
& D_{x}=\left\{H_{x i, j}, I_{x i, j}\right\} \\
& D_{y}=\left\{H_{y i, j}, I_{y i, j}\right\}
\end{aligned}
$$

Let

$$
G_{*}=\min _{(x, y) \in \bar{\Omega}} \frac{\partial f}{\partial U}, \quad G^{*}=\max _{(x, y) \in \bar{\Omega}} \frac{\partial f}{\partial U}
$$

where $\bar{\Omega}=\partial \Omega \cup \Omega$. Then

$$
0<G_{*} \leq t \leq G^{*} \quad, \forall \quad t \in G_{0}
$$

Let us assume that

$$
0<|\theta|<q_{k}, \quad q_{k}>0, \quad k=1(1) 4 \quad, \forall \quad \theta \in H_{0}, I_{0}, D_{x}, D_{y} .
$$


It is easy to verify for sufficiently small $h$ that

$$
\begin{array}{rll}
\left|P_{(j-1)(N-1)+i,(j-1)(N-1)+i}\right|<20, & {[i=1(1) N-1,} & j=1(1) N-1] . \\
\left|P_{(j-1)(N-1)+i,(j-1)(N-1)+i+1}\right|<4, & {[i=1(1) N-2,} & j=1(1) N-1] . \\
\left|P_{(j-1)(N-1)+i,(j-1)(N-1)+i-1}\right|<4, & {[i=2(1) N-1,} & j=1(1) N-1] . \\
\left|P_{(j-1)(N-1)+i,(j-2)(N-1)+i}\right|<4, & {[i=1(1) N-1,} & j=2(1) N-1] . \\
\left|P_{(j-1)(N-1)+i, j(N-1)+i}\right|<4, & {[i=1(1) N-1,} & j=1(1) N-2] . \\
\left|P_{(j-1)(N-1)+i,(j-2)(N-1)+i+1}\right|<1, & {[i=1(1) N-2,} & j=2(1) N-1] . \\
\left|P_{(j-1)(N-1)+i,(j-2)(N-1)+i-1}\right|<1, & {[i=2(1) N-1,} & j=2(1) N-1] . \\
\left|P_{(j-1)(N-1)+i, j(N-1)+i+1}\right|<1, & {[i=1(1) N-2,} & j=1(1) N-2] . \\
\left|P_{(j-1)(N-1)+i, j(N-1)+i-1}\right|<1, & {[i=2(1) N-1,} & j=1(1) N-2] .
\end{array}
$$

Let $R_{k}$ denote the sum of the elements in the $k^{\text {th }}$ row of the matrix $\mathbf{D}+\mathbf{P}=\mathbf{J}$. So for $k=1$ and $k=N-1$, we have

$$
\begin{aligned}
& R_{k}=11+\frac{h^{2}}{4}\left[28 G-4\left(H_{x}+I_{y}\right)+\left(2 H^{1}-H^{2}\right)(2 H-I)+\left(2 I^{1}-I^{2}\right)(2 I-H)\right. \\
& \left.+2\left(I_{x}+H_{y}\right)\right]+h\left(H+I+3\left(H^{1}+I^{1}\right)-\left(H^{2}+I^{2}\right)\right) . \\
& R_{(N-2)(N-1)+k}=11+\frac{h^{2}}{4}\left[28 G-4\left(H_{x}+I_{y}\right)+\left(2 H^{1}-H^{2}\right)(2 H-I)+\left(2 I^{1}-I^{2}\right)(2 I-H)\right. \\
& \left.+2\left(I_{x}+H_{y}\right)\right]-h\left(H+I+3\left(H^{1}+I^{1}\right)-\left(H^{2}+I^{2}\right)\right) .
\end{aligned}
$$

For $q=2(1) N-2$

$$
\begin{gathered}
R_{(q-1)(N-1)+1}=6+\frac{h^{2}}{2}\left(15 G+H\left(2 H^{1}-H^{2}\right)-2 H_{x}\right)+\frac{h}{2}\left(3 H+8 h^{1}-3 H^{2}\right) . \\
R_{q(N-1)}=6+\frac{h^{2}}{2}\left(15 G+H\left(2 H^{1}-H^{2}\right)-2 H_{x}\right)-\frac{h}{2}\left(3 H+8 H^{1}-3 H^{2}\right) .
\end{gathered}
$$

For $r=2(1) N-2$

$$
\begin{aligned}
R_{r} & =6+\frac{h^{2}}{2}\left(15 G+I\left(2 I^{1}-I^{2}\right)-2 I_{y}\right)-\frac{h}{2}\left(3 I+8 I^{1}-3 I^{2}\right) . \\
R_{(N-2)(N-1)+r} & =6+\frac{h^{2}}{2}\left(15 G+I\left(2 I^{1}-I^{2}\right)-2 I_{y}\right)+\frac{h}{2}\left(3 I+8 I^{1}-3 I^{2}\right) .
\end{aligned}
$$

For $q=2(1) N-2$ and $r=2(1) N-2$

$$
R_{(r-2)(N-1)+q}=8 h^{2}(G)
$$

Let $\mathbf{R}=\left(R_{1}, R_{2}, \ldots \ldots, R_{(N-1)^{2}}\right)^{t}$ denotes the row sums of $\mathbf{J}=\left(j_{i, j}\right)_{(N-1)^{2} \times(N-1)^{2}}$. It is easy to see that $\mathbf{J}$ is irreducible [7]. By the row sum criterion, for sufficiently small $h$, it follows that $\mathbf{J}$ is monotone $\left[8\right.$. Thus $(\mathbf{J})^{-1}$ exist. For bounds of $(\mathbf{J})^{-1}$, let define [9, 10,

$$
R_{i}(\mathbf{J})=2\left(\left|j_{i, i}\right|\right)-\sum_{i=1}^{(N-1)^{2}}\left|j_{i, j}\right|, \quad j=1,2, \ldots \ldots .,(N-1)^{2} .
$$

and

$$
R_{*}(\mathbf{J})=\min _{1 \leq i \leq(N-1)^{2}} R_{i}(\mathbf{J})
$$

Let us assume that $R_{i}(\mathbf{J}) \geq 0$ then

$$
\left\|\mathbf{J}^{-1}\right\|_{\infty} \leq \frac{1}{R_{*}(\mathbf{J})}
$$


Thus from (41) and (49), we have

$$
\|\mathbf{E}\|_{\infty} \leq \frac{1}{R_{*}(\mathbf{J})}\|\mathbf{T}\|_{\infty} .
$$

With the help of (33) and (49), for sufficiently small $h$, we have

$$
\|\mathbf{E}\| \leq O\left(h^{4}\right)
$$

Thus the proposed difference method (31) converges and the order of the convergence is four.

\section{Numerical Experiments}

The validity and effectiveness of the proposed exponential method on uniform mesh have been tested on linear and nonlinear model problems. We have considered a nonlinear Poisson equation, linear Poisson equation, nonlinear convection equation and problem governed by N-S in steady state condition in Cartesian coordinate system regardless the magnitude of the Reynold number. In each case we took a square as a region of integration and covered it with a uniform mesh of size h. We have used iterative method Gauss Seidel, Newton-Raphson to solve the system of linear and nonlinear equations respectively. In tables, we have shown maximum absolute error MAU, computed for different values of $\mathrm{N}$, using following formula,

$$
M A U=\max _{2 \leq i, j \leq N}\left|u\left(x_{i}, y_{j}\right)-u_{i, j}\right|
$$

For shake of comparison we presented the results from the published literature in the table. All the computations in the experiment were performed on MS Window 2007 professional operating system in the GNU FORTRAN environment version -99 compiler(2.95 of gcc) running on Intel Duo core 2.20 Ghz PC . The stopping condition for iteration was either error of order $10^{-10}$ or number of iterations $10^{3} \cdot$

Problem 1. Consider a nonlinear problem which, when solving consists of

$$
\frac{\partial^{2} u}{\partial x^{2}}+\frac{\partial^{2} u}{\partial y^{2}}=u\left(\frac{\partial u}{\partial x}+\frac{\partial u}{\partial x}\right)+f(x, y), \quad 0 \leq x, y \leq 1
$$

with the boundary conditions $u(x, y)$ on all sides of unit square. $f(x, y)$ is given such that the exact solution is $u(x, y)=\exp (-x) \cos (y)$. In tables 1 and 2 , we have presented the computed MAU for different values of $\mathrm{N}$ and different constructed solutions. The results in tables computed by same method but show the variation in MAU.

Problem 2. Consider the problem which, when solving consists of

$$
\frac{\partial^{2} u}{\partial x^{2}}+\frac{\partial^{2} u}{\partial y^{2}}=\frac{\partial u}{\partial x}+\frac{\partial u}{\partial y}+f(x, y), \quad 0 \leq x, y \leq 1
$$

with the boundary conditions $u(x, y)$ on all sides of unit square. $f(x, y)$ is given such that the exact solution is $u(x, y)=\sin (\pi x) \sin (\pi y)$. In table 3, we have presented the computed MAU for different values of $\mathrm{N}$.

Problem 3. Consider the problem of the nonlinear convection equation [6] which,when solving consists of

$$
\varepsilon\left(\frac{\partial^{2} u}{\partial x^{2}}+\frac{\partial^{2} u}{\partial y^{2}}\right)=u\left(\frac{\partial u}{\partial x}+\frac{\partial u}{\partial y}\right)+f(x, y), \quad 0 \leq x, y \leq 1
$$

with the boundary conditions $u(x, y)$ on all sides of unit square. $f(x, y)$ is given such that the exact solution is $u(x, y)=\exp (x) \sin ((\pi y) / 2)$. In tables 4 and 5 , we have presented the computed MAU for different values of $N$ and $\varepsilon$. In table, it shows results from [6], for shake of comparison.

Problem 4. Consider the problem of the nonlinear Navier-Stokes in steady state [6] which, when solving consists of

$$
\begin{aligned}
& \frac{1}{R e}\left(\frac{\partial^{2} u}{\partial x^{2}}+\frac{\partial^{2} u}{\partial y^{2}}\right)=u \frac{\partial u}{\partial x}+v \frac{\partial u}{\partial y}+f(x, y) \\
& \frac{1}{R e}\left(\frac{\partial^{2} v}{\partial x^{2}}+\frac{\partial^{2} v}{\partial y^{2}}\right)=u \frac{\partial v}{\partial x}+v \frac{\partial v}{\partial y}+g(x, y), \quad 0 \leq x, y \leq 1
\end{aligned}
$$


with the boundary conditions $u(x, y)$ and $v(x, y)$ are on all sides of unit square. $f(x, y)$ and $g(x, y)$ are given such that the exact solution are $u(x, y)=\sin (\pi x) \sin (\pi y)$ and $v(x, y)=\cos (\pi x) \cos (\pi y)$. In table 5 , we have presented the computed value of MAU and MAV for different values of $\mathrm{N}$ and Reynold number Re. In table, it shows results from [6], for shake of comparison.

Table 1: Maximum absolute errors in $u(x, y)=\exp (-x) \cos (y)$ for problem 1 .

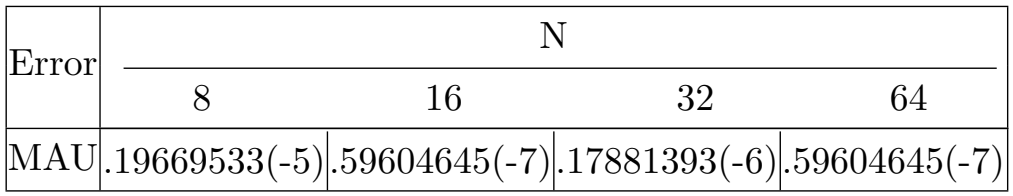

Table 2: Maximum absolute errors in $u(x, y)=\exp (x) \sin ((\pi y) / 2)$ for problem 1.

\begin{tabular}{|c|c|c|c|c|}
\hline \multirow{2}{*}{ Error } & \multicolumn{4}{|c|}{$N$} \\
\hline & 8 & 16 & 32 & 64 \\
\hline MAU & .17166138(-4) & $.59604645(-6)$ & $.23841858(-6)$ & $.47483716(-6)$ \\
\hline
\end{tabular}

Table 3: Maximum absolute in $u(x, y)=\sin (\pi x) \sin (\pi y)$ for problem 2 .

\begin{tabular}{|c|c|c|c|c|}
\hline \multirow{2}{*}{ Error } & \multicolumn{4}{|c|}{$\mathrm{N}$} \\
\hline & 16 & 32 & 64 & 128 \\
\hline MAU & $.15661716(-2) \cdot 33712387(-3)$ & $.79751015(-4)$. & $11622906(-4)$ & $.17881393(-6)$ \\
\hline
\end{tabular}

Table 4: Maximum absolute errors in $u(x, y)=\exp (x) \sin ((\pi y) / 2)$ for problem 3 .

\begin{tabular}{|c|cc|cc|cr|}
\hline \multirow{2}{*}{$\mathrm{N}$} & \multicolumn{7}{|c|}{ MAU } \\
\cline { 2 - 7 } & \multicolumn{2}{|c|}{$\varepsilon=0.1$} & \multicolumn{2}{|c|}{$\varepsilon=.01$} & \multicolumn{2}{c|}{$\varepsilon=.001$} \\
\hline 16 & $.13551399(-1)$ & $.20616(-4)$ & $.11508358(0)$ & $.24785(-3)$ & $.13903845(-1)$ & $.2513(-2)$ \\
\hline 32 & $.61949044(-2)$ & $.12695(-5)$ & $.36507472(-3)$ & $.17462(-4)$ & $.70639327(-2)$ & $.14391(-3)$ \\
\hline 64 & $.69573149(-3)$ & $.79012(-7)$ & $.30447170(-3)$ & $.11296(-5)$ & $.38139522(-4)$ & $.10375(-4)$ \\
\hline 128 & $.16582198(-4)$ & $* * * * *$ & $.10848977(-4)$ & $* * * * *$ & $.32613985(-4)$ & $* * * * *$ \\
\hline 256 & $.60684979(-5)$ & $* * * * *$ & $.55702403(-5)$ & $* * * * *$ & $.73881820(-5)$ & $* * * * *$ \\
\hline
\end{tabular}

$* * * * *$ : Computational results either over flow or not available.

Table 5: Maximum absolute errors in $u(x, y)=\exp (x) \sin ((\pi y) / 2)$ for problem 3 .

\begin{tabular}{|c|ccccc|}
\hline \multirow{2}{*}{ Error } & \multicolumn{5}{|c|}{$\varepsilon=.0001, N$} \\
\cline { 2 - 6 } & 16 & 32 & 64 & 128 & 256 \\
\hline MAU & $.40043145(-2)$ & $.51295012(-3)$ & $.58542937(-4)$ & $.13077632(-4)$ & $.19995435(-5)$ \\
\hline 6$]$ & $* * * * *$ & $* * * * *$ & $* * * * *$ & $* * * * *$ & $* * * * *$ \\
\hline
\end{tabular}


Table 6: Maximum absolute errors in $u(x, y)=\sin (\pi x) \sin (\pi y)$ and $v(x, y)=\cos (\pi x) \cos (\pi y)$ for problem 4 .

\begin{tabular}{|c|c|c|c|c|c|c|c|}
\hline \multirow{3}{*}{$\mathrm{N}$} & & \multicolumn{6}{|c|}{ Maximum absolute error } \\
\hline & & \multicolumn{2}{|c|}{$R e=1.0$} & \multicolumn{2}{|c|}{$R e=10$} & \multicolumn{2}{|c|}{$R e=100$} \\
\hline & & $(31)$ & 6 & $(31)$ & 6 & $(31)$ & 6 \\
\hline \multirow{2}{*}{8} & $\mathrm{u}$ & $.56552887(-2)$ & $* * * * *$ & $.62737405(-1)$ & $* * * * *$ & $.32716489(+1)$ & $* * * * *$ \\
\hline & $\mathrm{v}$ & $.41908026(-3)$ & $* * * * *$ & $.40605068(-2)$ & $* * * * *$ & $.63114434(0)$ & $* * * * *$ \\
\hline \multirow{2}{*}{16} & $\mathrm{u}$ & $.55550933(-2)$ & $* * * * *$ & $.16453490(-3)$ & $.38170(-4)$ & $.75181895(-1)$ & $.79117(-3)$ \\
\hline & $\mathrm{v}$ & $.37270784(-3)$ & $* * * * *$ & $.11771917(-3)$ & $.20205(-4)$ & $.62419891(-1)$ & $.81179(-3)$ \\
\hline \multirow{2}{*}{32} & $\mathrm{u}$ & $.55446029(-2)$ & $* * * * *$ & $.22990629(-4)$ & $.24148(-5)$ & $.33464283(-4)$ & $.44070(-4)$ \\
\hline & $\mathrm{V}$ & $.36579370(-3)$ & $* * * * *$ & $.28610229(-5)$ & $.12680(-5)$ & $.35881996(-4)$ & $.46982(-4)$ \\
\hline \multirow{2}{*}{64} & $\mathrm{u}$ & $.42155385(-4)$ & $* * * * *$ & $.50500967(-6)$ & $.15149(-6)$ & $.10032672(-5)$ & $.26562(-5)$ \\
\hline & $\mathrm{V}$ & $.12695789(-4)$ & $* * * * *$ & $.11920929(-6)$ & $.79504(-7)$ & $.71525574(-6)$ & $.30175(-5)$ \\
\hline \multirow{2}{*}{128} & $\mathrm{u}$ & $.24447218(-8)$ & $* * * * *$ & $.39231963(-7)$ & $* * * * *$ & $.24237670(-6)$ & $* * * * *$ \\
\hline & $\mathrm{v}$ & $.59604645(-7)$ & $* * * * *$ & $.59604645(-7)$ & $* * * * *$ & $.17881393(-6)$ & $* * * * *$ \\
\hline \multirow{2}{*}{256} & $\mathrm{u}$ & $* * * * *$ & $* * * * *$ & $.87311491(-9)$ & $* * * * *$ & $.36816346(-8)$ & $* * * * *$ \\
\hline & $\mathrm{V}$ & $* * * * *$ & $* * * * *$ & $.59604645(-7)$ & $* * * * *$ & $.59604645(-7)$ & $* * * * *$ \\
\hline
\end{tabular}

$* * * * *$ : Computational results either over flow or not available.

\section{Conclusion}

In this article we have applied exponential difference method for numerical solution of general nonlinear elliptic equations. The method is fourth order accurate and Dirichlet boundary conditions incorporated into the method. To bring out different aspect of the method, we employed it to compute the numerical solution of steady state Navier Stokes equation and convection equation with their constructed exact solutions. For shake of comparison, we have done with methods in 5, 6 and find that present method compare favorably. In one considered model problem we find that the computational performance of the method and accuracy depends on the constructed exact solution. The results obtained in model problems are in good agreement to the estimated order of the method. It is observed from the results that method has higher accuracy i.e. small discretization error. The numerical experiments show that this proposed exponential method has a good numerical stability in considered examples. Though method developed and used on rectangular domains, it has good potential for efficient application to many problems on different geometries, work in this specific direction is in progress.

\section{References}

[1] H. Johansen, and P. Colella, A Cartesian grid embedded boundary method for Poisson's equation on irregular domains. J. Comput. Phys., Vol. 147(2),60-85 (1998).

[2] M. M. Gupta, R. P. Manohar, and J. W. Stephenson, A single cell high order scheme for the convection-diffusion equation with variable coefficients.Int. J. Numer. Methods in Fluid 4,641-651(1984).

[3] G.Saldanha, and U. Ananthakrishnaiah, A fourth-order finite difference scheme for two-dimensional nonlinear elliptic partial differential equations. Numer. Meth. Partial Diff. Eqns. 11,33 - 40(1995). 
[4] R. K. Mohanty, Order $h^{4}$ difference methods for a class of singular two space elliptic boundary value problems. J. Comp. Appl. Math. 81, 229-247 (1997).

[5] P. K. Pandey, A higher Accuracy exponential Finite Difference Method for Numerical Solution of Second Order Elliptic Partial Differential Equations. J. Math. Comput. Sci. 3, No.5, 1325 - 1334 (2013).

[6] R. K. Mohanty, and N. Setia, A New Fourth-Order Compact Off-Step Discretization for the System of 2D Nonlinear Elliptic Partial Differential Equations.East Asian Journal on Applied Mathematics Vol.2, No. 1, pp. 59-82 (2012).

[7] R. S. Varga, Matrix Iterative Analysis, Second Revised and Expanded Edition, Springer-Verlag, Heidelberg, (2000).

[8] P. Henrici, Discrete Variable Methods in Ordinary Differential Equations, John Wiley and Sons, New York, (1962).

[9] Y. S. Volkov, and V. L. Miroshnichenko, Norm estimates for the inverses of matrices of monotone type and totally positive matrics. Siberian Mathematical Journal Vol. 50, No. 6, pp. 982-987(2009).

[10] J. H. Ahlberg, and E. N. Nilson, Convergence properties of the spline fit. J. SIAM, 11, No. 1, 95-104 (1963). 